\title{
The unification of inflation and the late-time acceleration
}

\section{Rio Saitou*}

Nagoya University

E-mail: saidth.phys.nagova-u.ac.jp

\section{Shin'ichi Nojiri}

Nagoya University, Kobayashi-Maskawa Institute for the Origin of Particles and the Universe E-mail: nojiridphys.nagoya-u.ac.jp

By using the formulation of the reconstruction, we explicitly construct models of k-essence, which unify the inflation in the early universe and the late accelerating expansion of the present universe by a single scalar field. Due to the higher derivative terms, the solution describing the unification can be stable in the space of solutions, which makes the restriction for the initial condition relaxed. Therefore we can construct a model describing the time development, which cannot be realized by a usual inflaton or quintessence models of the canonical scalar field due to the instability. We also propose a mechanism of the reheating by the quantum effects coming from the variation of the energy density of the scalar field.

KMI International Symposium 2013 on "Quest for the Origin of Particles and the Universe", 11-13 December, 2013

Nagoya University, Japan

\footnotetext{
* Speaker.
} 


\section{Introduction}

We now believe the accelerating expansion of the present universe by several cosmological

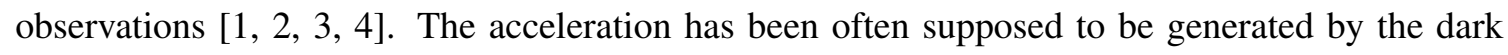
energy which is an unknown fluid. So-called $k$-essence model [[5] is a model of the dark energy. The $k$-essence model is originated from $k$-inflation model [ 6$]$.

From the above, it might be natural to consider a model unifying the inflation and the late

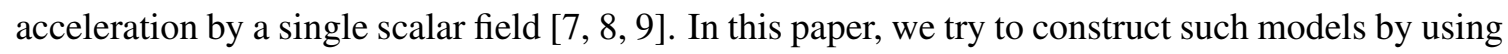
the formulation of the reconstruction [एव, प] and we also propose a mechanism of the reheating by the quantum effects. In the models, the solution which describes the unification of the inflation and the late acceleration can be stable and the attractor in the space of solutions and also the propagating mode around the solution does not violate causality due to the higher derivative terms.

\section{Reconstruction}

We consider the following action;

$$
S=\int d^{4} x \sqrt{-g}\left(\frac{R}{2 \kappa^{2}}-K(\phi, X)+L_{\text {matter }}\right), \quad X \equiv \partial^{\mu} \phi \partial_{\mu} \phi .
$$

Here $\phi$ is a single scalar field and $K(\phi, X)$ is a general function of $\phi$ and $X$ called k-essence. We assume flat homogeneous and isotropic space-time and also that there is no direct interaction between ordinary matter and k-essence effectively except the reheating era. Then by using the appropriate function $g_{\phi}(\phi)$, if we choose

$$
\begin{aligned}
& K(\phi, X)=\sum_{n=0}^{\infty}\left(\frac{X}{g_{\phi}(\phi)+\frac{\kappa^{2}}{3} \rho_{\text {matter }}(\phi)}+1\right)^{n} \tilde{K}^{(n)}(\phi), \\
& \tilde{K}^{(0)}(\phi) \equiv-\frac{1}{\kappa^{2}}\left(g_{\phi}^{\prime}(\phi)+3 g_{\phi}(\phi)\right), \quad \tilde{K}^{(1)}(\phi)=\frac{1}{2 \kappa^{2}} g_{\phi}^{\prime}(\phi),
\end{aligned}
$$

we find the following solution of the Friedmann equations derived from the action (‥D) which could be valid except the reheating era:

$$
G(N)=H(N)^{2}=g_{\phi}(N)+\frac{\kappa^{2}}{3} \rho_{\text {matter }}(N), \quad \phi=N \quad\left(X=-H^{2}\right),
$$

where $N \equiv \ln a / a_{0}$ is the e-folding number which we use as a clock.

In order that the solution $([2.3])$ is stable and keep causality, we consider the linear perturbations of the solution (2.3]) and of the background metric respectively. Then, we get the constraints for $\tilde{K}^{(1)}(N)$ and $\tilde{K}^{(2)}(N)$ demanding that the perturbation from the solution must converge for all $N$ and that the perturbation of the metric must not propagate faster than the light speed;

$$
\begin{aligned}
& 3>\frac{G_{0}^{\prime}(N)}{2 G_{0}(N)}, \\
& H^{2} \tilde{K}^{(1) \prime}(N)-2 H H^{\prime} \tilde{K}^{(1)}(N)+3 \kappa^{2} \tilde{K}^{(1)}(N) \tilde{K}^{(1) \prime}(N)+2\left(\tilde{K}^{(1)}(N)\right)^{2} \\
& +\left(4 H H^{\prime}-12 \kappa^{2} \tilde{K}^{(1) \prime}(N)-4 \kappa^{2} \tilde{K}^{(1)}(N)\right) \tilde{K}^{(2)}(N)>0, \\
& \tilde{K}^{(2)}(N)>0 \text { or } \tilde{K}^{(2)}(N)<\frac{1}{4} \tilde{K}^{(1)} .
\end{aligned}
$$




\section{Unifying model}

When constructing the model which unify inflation and late-time acceleration, we assume the following: 1) The energy scale of inflation should be almost equal to the GUT scale. 2) Except the period of the particle production, the EoS parameter $w_{\phi}$ for the scalar field $\phi$ could be given by $w_{\phi}(N)=-1-\frac{\rho_{\phi}^{\prime}(N)}{3 \rho_{\phi}(N)}$. 3) In general, there are two solutions $N=N_{1}, N_{2}\left(N_{1}<N_{2}\right)$ in the equation $\frac{\left.\rho_{\phi}^{\prime}\right|_{N=N_{1,2}}}{\left.\rho_{\phi}^{\prime}\right|_{\max }}=\frac{1}{\mathrm{e}}$. We expect that the expression of $w_{\phi}$ could become invalid when $N_{1}<N<N_{2} .4$ ) The inflation started at $N=0$ and the end of the inflation is defined by $N=N_{\mathrm{I}} \equiv N_{1} \simeq 60$. 5) The reheating and the particle production could have occurred when $N_{1} \lesssim N \lesssim N_{2}$. 6) The reheating temperature $T_{\mathrm{RH}}$ could be $10 \mathrm{MeV}<T_{\mathrm{RH}}<10^{14} \mathrm{GeV}$.

Furthermore, the cosmological observations tell, at present $N_{0}$, 7) $\rho_{\phi}\left(N=N_{0}\right) \simeq 10^{-47} \mathrm{GeV}^{4}$, 8) $w_{\phi}=-1.023 \pm 0.144$ at $N_{0}$.

Then, as the appreciate function $\rho_{\phi}(N)=\frac{3}{\kappa^{2}} g_{\phi}(N)$ (equal to the energy density of k-essence), for example, we choose the following function :

$$
\rho_{\phi}(N)=M^{4} \exp \left(-\frac{1}{d^{-1}+c_{1} \exp \left(-\frac{N-N_{\mathrm{I}}}{\Delta_{1}}\right)}\right) .
$$

Here, $c_{1}, d$ and $\Delta_{1}$ are constants and we choose $M \simeq 10{ }^{15} \mathrm{GeV}$ and $c_{1} \simeq 6.309$. This function has the parameter region of $\Delta_{1}$ which satisfy all the above constraints 1$)$ - 8). In case $T_{\mathrm{RH}}=10 \mathrm{MeV}$, we find $(0,246.4)<\left(\Delta_{1}, d\right)<(1.81,247)$ as the suitable parameter region and in case $T_{\mathrm{RH}}=10^{14} \mathrm{GeV}$, $(0,246.4)<\left(\Delta_{1}, d\right)<(4.97,248.2)$.

Furthermore, we can find that there exists a regular and nonzero $\tilde{K}^{(2)}(\phi)$ which satisfy the constraints (2.4) for the above region of $\Delta_{1}$. Therefore, we constructed the stable model which unify inflation and late-time acceleration and does not violate causality due to the coefficient of higher order kinetic term, $\tilde{K}^{(2)}(\phi)$.

\section{A mechanism of particle production}

Now we assume the Hubble rate is given in terms of the e-folding $N$ as $H=H(N)$ and consider the situation that the e-folding $N$ can be identified with a scalar field $\phi$. We now consider the interaction between the scalar field $\phi$ between another real scalar field $\varphi$ as follows,

$$
H_{\text {int }}=-\frac{C_{0}}{2} \int d^{3} x \sqrt{-g} \frac{d \rho_{\phi}(\phi)}{d \phi} \varphi^{2} \sim \frac{U_{0}}{\Delta \sqrt{\pi}} \mathrm{e}^{-\frac{t^{2}}{\Delta^{2}}} .
$$

Here $C_{0}$ and $U_{0}$ is a constant and $\Delta$ is the standard deviation. Note that we treat the scalar field $\phi$ like a classical external source and $\phi$ 's classical energy in the coherent state excites $\varphi$ 's quantum state. We assume that the space-time can be regarded as static and also flat when $|t| \sim \Delta$, which should be checked.

Then the amplitude that the vacuum could transit to two-particle state whose momenta are given by $\mathbf{p}$ and $\mathbf{q}$ is given by

$$
A_{\mathbf{p q}}=i \int_{-\infty}^{\infty} d t\left\langle\mathbf{p}, \mathbf{q}\left|\mathbf{H}_{\text {int }}\right| \mathbf{0}\right\rangle
$$




$$
=i \frac{U_{0}}{\Delta \sqrt{\pi}} \int_{-\infty}^{\infty} d t \int d^{3} x \frac{\mathrm{e}^{-\frac{t^{2}}{\Delta^{2}}-i\left(\omega_{p}+\omega_{q}\right)+i(\mathbf{p}+\mathbf{q}) \cdot \mathbf{x}}}{2 \sqrt{\omega_{p} \omega_{q}}}=i \delta^{3}(\mathbf{p}+\mathbf{q}) \frac{U_{0} \mathrm{e}^{-\Delta^{2} \omega_{p}^{2}}}{2 \omega_{p}} .
$$

Here $\omega_{p}=\sqrt{\mathbf{p}^{\mathbf{2}}+\mathbf{m}_{\varphi}^{\mathbf{2}}}$ with the mass $m_{\varphi}$ of $\varphi$. When $\varphi$ is massless, from (4.2), we can calculate the expectation value of the energy density $\varepsilon_{2}$ corresponding to two-particle state as

$$
\varepsilon_{2}=\frac{1}{2 V} \int d^{3} p d^{3} q \delta^{3}(\mathbf{p}+\mathbf{q})^{2} \frac{2 \omega_{p} U_{0}^{2} \mathrm{e}^{-2 \Delta^{2} \omega_{p}^{2}}}{4 \omega_{p}^{2}}=\frac{U_{0}^{2}}{8(2 \pi)^{\frac{3}{2}} \Delta} .
$$

Here $V \equiv(2 \pi)^{3} \delta^{3}(0)$ is the volume of space. Then, we compare (4.3) with the energy density of the radiation at reheating era which can estimate from the present value of it, and finally we get the relation

$$
U_{0}=9.86 \Delta_{1} \times 10^{-15}-10^{17} \mathrm{GeV} \text { for } T_{\mathrm{RH}}=10 \mathrm{MeV}-10^{14} \mathrm{GeV} .
$$

\section{Summary}

In this proceeding, after formulating the reconstruction for $k$-essence with $N$, we explicitly constructed a model which unify the inflation in the early universe and the late-time acceleration in the present universe, and satisfy the observational constraints. We have proposed a mechanism of the interaction for particle production by the quantum effects coming from the variation of the energy density of the scalar field and estimated the energy density of the particles.

In the model, the solutions describing the development of the universe expansion are stabilized by $\tilde{K}^{(2)}$ term which should not vanish. We also note that $\tilde{K}^{(2)}$ term play the role to keep causality of the propagation of the metric perturbation.

\section{References}

[1] For instance, D. N. Spergel et al. [ WMAP Collaboration ], Astrophys. J. Suppl. 148, 175-194 (2003).

[2] E. Komatsu et al. [ WMAP Collaboration ], Astrophys. J. Suppl. 180, 330-376 (2009).

[3] For instance, S. Perlmutter et al. [ Supernova Cosmology Project Collaboration ], Astrophys. J. 517, 565-586 (1999).

[4] P. Astier et al. [ The SNLS Collaboration ], Astron. Astrophys. 447, $31-48$ (2006).

[5] T. Chiba, T. Okabe and M. Yamaguchi, Phys. Rev. D 62, 023511 (2000) .

[6] C. Armendariz-Picon, T. Damour and V. F. Mukhanov, Phys. Lett. B 458, 209 (1999) .

[7] P. J. E. Peebles, A. Vilenkin, Phys. Rev. D59, 063505 (1999).

[8] N. Bose, A. S. Majumdar, Phys. Rev. D80, 103508 (2009).

[9] J. De-Santiago, J. L. Cervantes-Cota, Phys. Rev. D83, 063502 (2011).

[10] S. Nojiri and S. D. Odintsov, J. Phys. Conf. Ser. 66, 012005 (2007).

[11] J. Matsumoto and S. Nojiri, Phys. Lett. B 687, 236 (2010) . 